\title{
Tsafon
}

Revue d'études juives du Nord

$76 \mid 2018$

Expressions yiddish de la nature

\section{Entraides, mémoires et migrations dans le monde yiddish. Une lettre de Yankev Pat à Marek Edelman, New York-Paris, 8 octobre 1946}

\section{Constance Pâris de Bollardière}

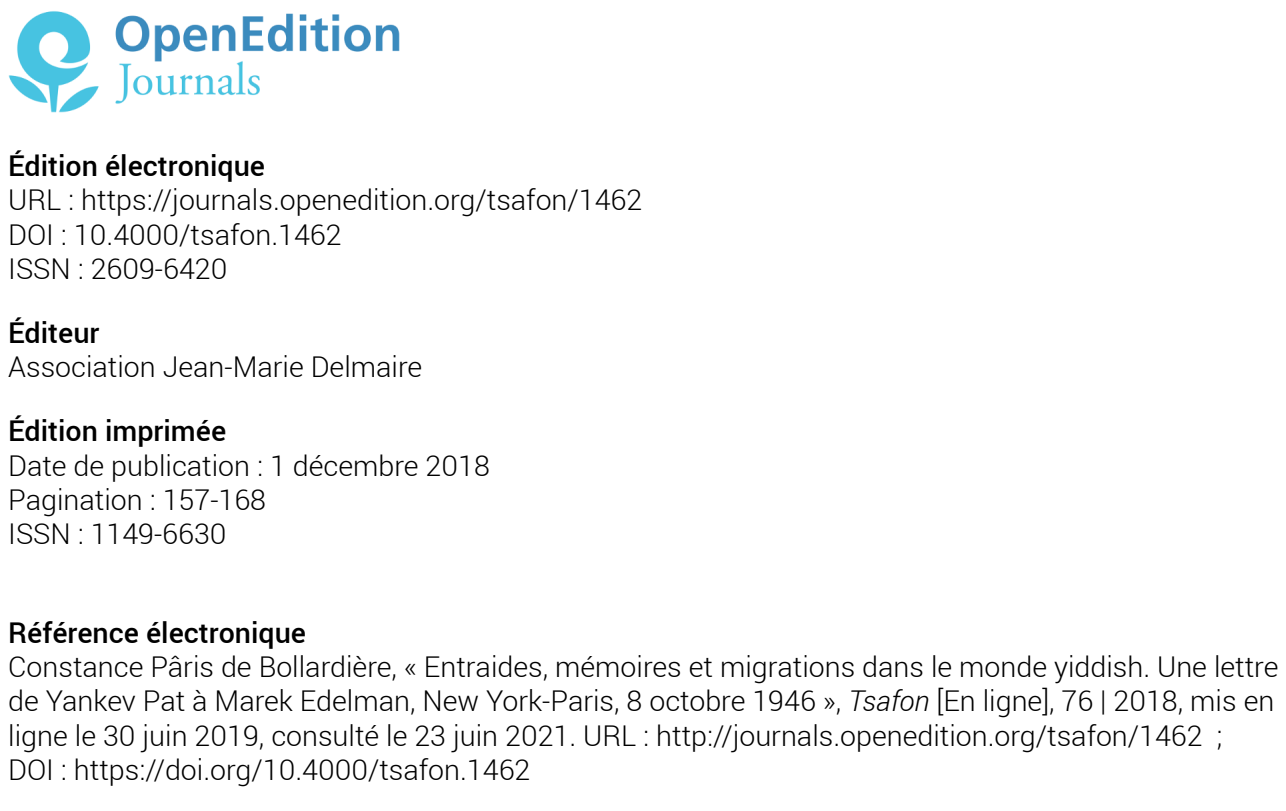

Tsafon. Revues d'études juives du Nord 


\title{
Édition
}

\section{Entraides, mémoires et migrations dans le monde yiddish}

\author{
Une lettre de Yankev Pat à Marek Edelman, \\ New York-Paris, 8 octobre 1946
}

\section{Constance Pâris de Bollardière*}

En octobre et novembre 1946, le jeune bundiste polonais Marek Edelman (1919-2009), ancien dirigeant de l'insurrection du ghetto de Varsovie, séjourna plusieurs semaines à Paris. C'est après s'être successivement rendu en Suède, en Belgique puis en Italie qu'Edelman et sa compagne Alina Margolis visitèrent la capitale française ${ }^{1}$. Grâce à des entretiens réalisés à la fin des années 2000, nous savons que ce dernier participa en Belgique à une conférence socialiste - au cours de laquelle il critiqua fermement l'inaction des partis sociaux-démocrates européens face à l'extermination des Juifs - et qu'il rencontra Léon Blum à Paris devant le palais du Luxembourg, entrevue lors de laquelle les deux socialistes échangèrent avec optimisme sur l'avenir de l'Europe ${ }^{2}$.

La lettre du 8 octobre 1946 que nous présentons dans cet article nous indique que, lors de son passage à Paris, Edelman entra aussi en contact avec les bundistes locaux comme avec ceux, américains, du Jewish Labor Committee. C'est des archives de ce comité qu'est extraite cette lettre ${ }^{3}$. Organisation ouvrière juive américaine anti-nazie, le Jewish

\footnotetext{
* American University of Paris.

${ }^{1}$ Witold Bereś et Krzysztof Burnetko, Marek Edelman. Życie. Po prostu, Varsovie, Świat Książki, 2008, p. 248. Je tiens à remercier Salomon Bielasiak pour sa traduction du polonais.

${ }^{2}$ Ibid., p. 249-250.

${ }^{3}$ Jewish Labor Committee Records, Tamiment Library, Wagner Labor Archives, New York University, Part I (1934-1947), WAG.025.001 (désormais JLC I), B 32 F 22.
} 
Labor Committee (JLC) fut créé à New York en 1934 par des bundistes ayant immigré aux États-Unis depuis l'Empire russe au lendemain de la révolution de 1905. Des décennies plus tard, alertés par l'arrivée au pouvoir d'Hitler, ces socialistes décidèrent de mobiliser le mouvement ouvrier juif et le mouvement ouvrier américain dans une lutte commune contre le fascisme et le nazisme. En plus de ces activités politiques, le JLC mena avant, pendant et après la Seconde Guerre mondiale une action d'aide aux victimes juives et non juives du nazisme qui étaient proches de sa mouvance politique ou de son univers culturel yiddishophone. Marqué par ses racines bundistes, l'organisation développa ainsi une relation privilégiée avec les rescapés juifs d'Europe issus de ce mouvement ${ }^{4}$.

La lettre que le secrétaire général du JLC, Yankev Pat, adressa à Marek Edelman le 8 octobre 1946 ne nous renseigne que marginalement sur le séjour parisien du dirigeant de l'insurrection du ghetto de Varsovie. Cet échange entre les deux bundistes illustre en revanche plusieurs facettes de l'action du JLC et de Yankev Pat en faveur de la mémoire du monde juif exterminé et de la reconstruction de la vie juive en France et en Pologne. Une part importante de cette entreprise concerna notamment l'assistance aux milliers de Juifs polonais fuyant l'antisémitisme en direction de l'Europe occidentale, préoccupation majeure du monde juif parisien et des organisations juives américaines de secours à la fin des années $1940^{5}$. Candidat potentiel à cette émigration, Marek Edelman est ici encouragé par Yankev Pat à quitter la Pologne, incitation révélant les dilemmes du dirigeant du JLC quant aux efforts que son organisation investissait dans l'avenir de la vie juive dans ce pays.

\footnotetext{
${ }^{4}$ Gail Malmgreen, «Labor and the Holocaust: The Jewish Labor Committee and the Anti-Nazi Struggle », Labor's Heritage 3 (4), 1991, p. 21-35; Catherine Collomp, Résister au nazisme, le Jewish Labor Committee, New York, 1934-1944, Paris, CNRS Éditions, 2016 ; Constance Pâris de Bollardière, "'La pérennité de notre peuple' : une aide socialiste juive américaine dans la diaspora yiddish, le Jewish Labor Committee en France (1944-1948) », thèse de doctorat d'histoire, École des Hautes Études en Sciences Sociales, Paris, 2017.

${ }^{5}$ L'historien David Weinberg parle de 80000 Juifs d'Europe de l'Est arrivés en France entre fin 1946 et début 1948, cf. David H. Weinberg, «A Forgotten Postwar Jewish Migration : East European Jewish Refugees and Immigrants in France, 1946-1947 », dans Françoise Ouzan et Manfred Gerstenfeld (éd.), Postwar Jewish Displacement and Rebirth, 1945-1967, Leyde/Boston, Brill, 2014, p. 137. Les démographes Sergio Della Pergola et Doris Bensimon estiment eux que 32000 Juifs immigrés ne provenant pas d'Afrique du Nord arrivent en France entre 1944 et 1949, cf. La population juive de France: socio-démographie et identité, Jérusalem/Paris, Hebrew University of Jerusalem/CNRS, 1986, p. 36.
} 


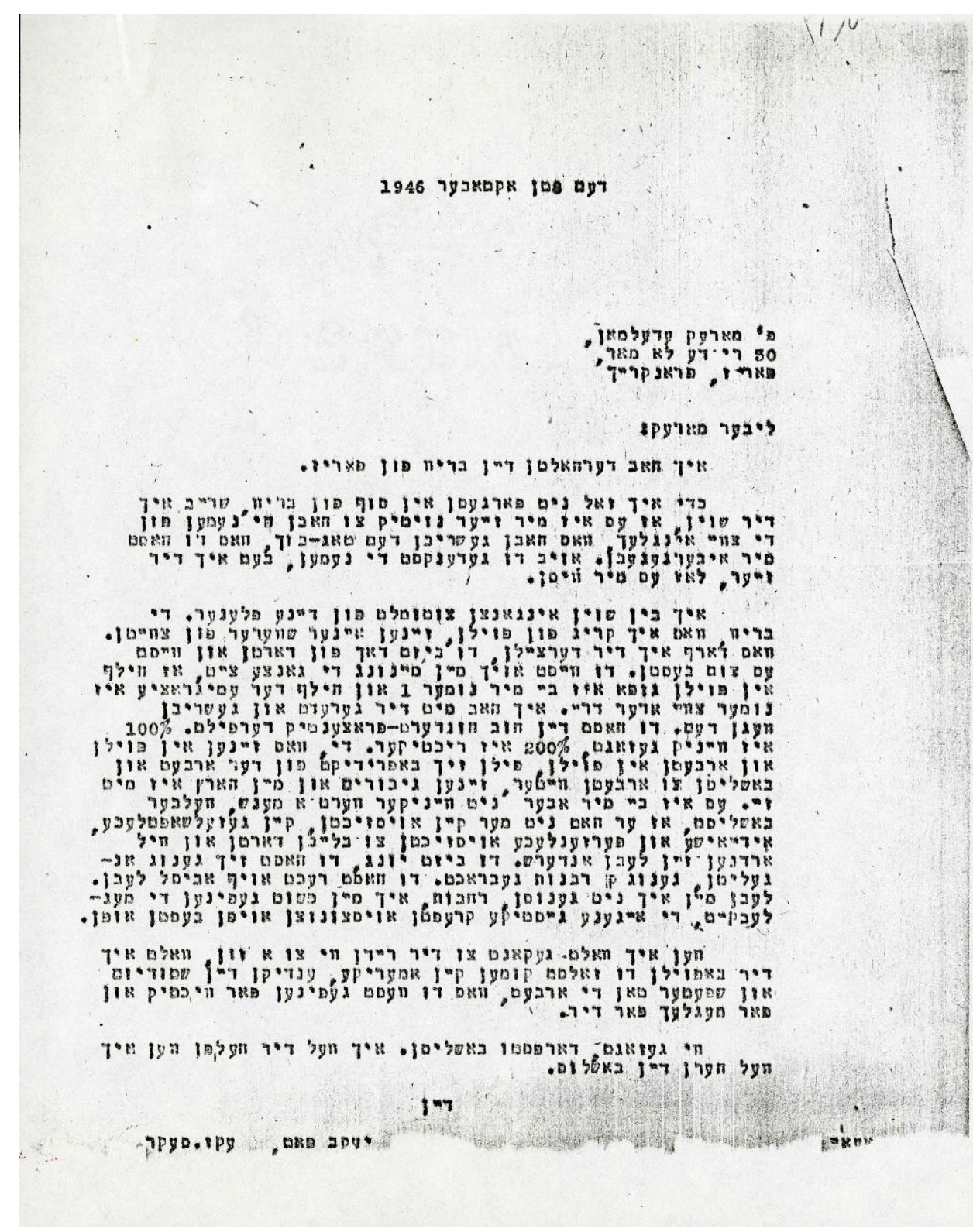


Traduction en français par Constance Pâris de Bollardière et Erez Lévy

Le 8 octobre 1946

M. Marek Edelman,

30 rue de la Mare,

Paris, France

Cher Marek :

J'ai bien reçu ta lettre de Paris.

Afin que je n'oublie pas à la fin de la lettre, je t'écris pour commencer qu'il est de la plus haute importance pour moi d'avoir les noms des deux jeunes qui ont écrit le journal de bord que tu m'as remis. Si tu te souviens de leurs noms, je t'en prie, fais-le-moi savoir.

Je suis complètement bouleversé par tes projets. Les lettres que je reçois de Pologne sont toutes plus dures les unes que les autres. Que puis-je te dire ? Tu es toi-même de là-bas et le sais parfaitement. Tu connais aussi mon opinion depuis le début, à savoir que l'aide en Pologne passe pour moi en premier et l'aide à l'émigration passe en deuxième ou troisième priorités. J'en ai déjà discuté avec toi et t'ai écrit à ce sujet. Tu as rempli ta mission à cent pour cent. $100 \%$ est peu dire, $200 \%$ serait plus exact. Ceux qui se trouvent en Pologne et travaillent en Pologne, trouvent du contentement à ce travail et choisissent de le poursuivre, sont des héros et je suis de tout cœur avec eux. Mais selon moi un homme n'a pas moins de valeur s'il décide qu'il n'a plus de perspectives, ni dans la vie associative, ni du point de vue des idéaux ou d'une vie personnelle en restant là-bas et souhaite faire sa vie ailleurs. Tu es jeune, tu as assez souffert, tu as fait assez de sacrifices. Tu as le droit de vivre un peu. Par vivre je n'entends pas jouissances et confort mais simplement trouver la possibilité d'exploiter ses propres forces spirituelles de la meilleure manière qu'il soit.

Si je pouvais te parler comme à un fils, je te conseillerais fortement de venir en Amérique, de finir tes études et de faire par la suite le travail que tu estimeras être important et possible pour toi.

Comme je te l'ai dit, ce choix t'appartient et quand tu m'en feras part, tu pourras compter sur mon aide.

Bien à toi,

Yankev Pat, secr. ex. 


\section{Yankev Pat, le Jewish Labor Committee et la reconstruction de la vie juive après-guerre}

Avant de développer les enjeux figurant au cœur de cette lettre, présentons son auteur Yankev Pat, bundiste, journaliste, éducateur, écrivain yiddish et acteur de la reconstruction de la vie juive. Né à Białystok en 1890 au sein d'une famille de condition modeste, Yankev Pat abandonna à la veille de la révolution russe de 1905 la formation religieuse qu'il suivait dans les yeshives (établissements d'études talmudiques supérieures) de Slabodka puis de Sloutsk et rejoignit le mouvement révolutionnaire juif. Si sa première famille politique fut le Poale Zion, parti sioniste marxiste au service duquel il exerça ses talents d'orateur à travers les bourgades juives de Pologne, il s'engagea à partir de 1920 dans les rangs du Bund, devint membre du comité central du parti et le représenta dans la kehile (communauté juive organisée) locale. Son engagement politique s'accompagna de fonctions dirigeantes dans les instances culturelles et éducatives yiddish de Pologne. Son expérience pédagogique débuta en 1915 à Białystok où Pat participa à la création de la première école yiddish, puis se poursuivit au lendemain de la Première Guerre mondiale à Vilnius où il enseigna, administra l'école Y. L. Peretz et devint secrétaire de la section locale de la Tsentrale yidishe shul organizatsye (Organisation centrale des écoles yiddish - TSYSHO). À Varsovie, où il passa la majorité de sa vie d'adulte d'avant-guerre, de 1922 à 1938, Pat poursuivit son double activisme politique et culturel. Il y dirigea le journal pour enfants du Bund, le Kleyne folkstsaytung (Petit journal populaire), ainsi que la section culturelle et éducative du Folkstsaytung (Journal populaire) de 1926 à 1938, devint secrétaire de la TSYSHO et représenta le Bund à la Literatn-fareyn (Union littéraire yiddish) située 13 rue Tłomackie. Parallèlement à ces activités politiques, culturelles et pédagogiques, Pat était un journaliste et écrivain pour adultes et enfants s'exprimant quasi exclusivement en langue yiddish. Arrivé à New York en 1938 lors d'une campagne de levée de fonds pour les écoles yiddish de Pologne, Pat y demeura après le déclenchement de la guerre et devint secrétaire-général du Jewish Labor Committee (JLC) en 1941, fonction qu'il conserva jusqu'en $1963^{6}$. Personnalité phare dans

\footnotetext{
${ }^{6}$ Zalman Rejzen, «Pat, Yankev », dans Id. (éd.), Leksikon fun der yidisher literatur, prese un filologye, Vilnius, Vilner Farlag, 1927, vol. 2, p. 850-854; Yeshayohu Shpigl, «Pat, Yankev », dans Efroym Oyerbakh et al. (éd.), Leksikon fun der nayer yidisher literatur, New York, Congress for Jewish Culture, 1968, vol. 7, p. 69-76 ; Emanuel Patt, In gerangl. Yankev Pat un zayn dor, New York, Yankev Pat Familye Fond, 1971. Sur
} 
les réseaux d'entraide bundiste après-guerre, Yankev Pat fut en charge du suivi du travail de reconstruction effectué par le JLC en Europe. En effet, en tant que bundiste influent dans la Pologne de l'entre-deux-guerres et qu'écrivain yiddish jouissant d'une certaine notoriété parmi les Juifs polonais yiddishophones, Pat était à la fois connu de nombreux rescapés de la Shoah que le JLC voulait aider tout en s'étant suffisamment intégré dans le mouvement ouvrier juif new-yorkais pour bâtir des ponts nécessaires à cette action transatlantique.

Centrée sur l'aide aux Juifs socialistes et yiddishophones originaires de Pologne et dans une moindre mesure de Roumanie, l'action du JLC à laquelle œuvra Pat ne constituait alors qu'une des ramifications du vaste programme d'aide juive internationale développé après la Shoah, qu'il s'agisse de l'aide juive américaine massive telle que celle de l'American Jewish Joint Distribution Committee ou de l'entraide appuyée sur des réseaux plus spécifiques du monde juif tels ceux des landsmanshaftn (sociétés d'originaires du monde juif d'Europe orientale $)^{7}$. Centralisant plusieurs réseaux non communistes du monde yiddish en tête desquels se trouvait le Bund, le JLC vint donc prioritairement en aide aux bundistes parisiens qui, depuis la Libération, reprenaient au grand jour leurs activités sociales, politiques et culturelles depuis le local de leur société de secours mutuel l'Arbeter-ring (Cercle ouvrier $^{8}$ situé 110 rue Vieille-du-Temple ${ }^{9}$ dans le Marais ou depuis son annexe du 30 rue de la Mare à Belleville, où fonctionnait leur dispensaire médical $^{10}$. C'est à cette dernière adresse que Pat adressa son courrier à Edelman en octobre 1946, preuve parmi d'autres que ce dernier fréquenta à plusieurs reprises le groupe de socialistes juifs parisiens ${ }^{11}$. Si nous ne

Yankev Pat et son aide à la reconstruction après-guerre, voir aussi Constance Pâris de Bollardière, "'Écritures de la destruction' et reconstruction : Yankev Pat, auteur et acteur du monde yiddish. Le cas de Paris, 1944-1948 », dans Judith Lindenberg (éd.), Premiers savoirs de la Shoah, Paris, CNRS Editions, 2017, p. 275-291.

7 Sur l'aide juive américaine, et en particulier celle de l'American Jewish Joint Distribution Committee, en France après-guerre, cf. Laura Hobson Faure, Un "Plan Marshall juif ». La présence juive américaine en France après la Shoah, 1944-1954, Paris, Armand Colin, 2013.

${ }^{8}$ La traduction française de l'époque était cependant « Cercle amical ».

${ }^{9}$ Cette adresse figure sur le tampon de la bibliothèque apposé sur la page de titre de l'ouvrage Henekh, reproduite dans cet article en illustration.

${ }^{10}$ Sur l'aide sociale Bund en France après-guerre, cf. Constance Pâris de Bollardière, «Mutualité, fraternité et travail social chez les bundistes de France, 1944-1947 », Archives Juives. Revue d'histoire des Juifs de France 45 (1), 2012, p. 27-42.

${ }^{11}$ Deux autres courriers des archives du JLC mentionnent les contacts entre Edelman et les bundistes à Paris : Pat à Edelman, 9 octobre 1946 et Khil Najman à Yankev Pat, 15 
savons pas quel fut le degré de proximité entre Edelman et ces derniers, cette lettre nous permet de constater que Pat et Edelman, qui s'étaient rencontrés quelques mois auparavant en Pologne et avaient depuis longuement échangé sur la situation des Juifs après-guerre («J'en ai déjà discuté avec toi et t'ai écrit à ce sujet»), avaient des rapports assez intimes pour se tutoyer ${ }^{12}$. Cette relation ne semble en revanche pas avoir assez marqué Marek Edelman pour que ce dernier nomme Yankev Pat des décennies plus tard, dans des entretiens réalisés au cours des années 2000, lors desquels il fit uniquement mention d'« un représentant du Jewish Labor Committee » venu en Pologne ${ }^{13}$.

\section{La publication du journal du jeune Henekh}

Au début de l'année 1946, Yankev Pat retourna en effet dans son pays natal afin d'y constater les besoins des rescapés juifs et de mettre en place une aide américaine appropriée. Deux ouvrages sont issus de ce séjour. Le premier, Ash un fayer (Cendres et feu) relate sa visite auprès des Juifs rescapés dans différentes villes de Pologne et décrit le sort des Juifs pendant la guerre ainsi que leurs efforts de reconstruction dans un pays en ruines ${ }^{14}$. Lors de son périple à travers les villes et bourgades polonaises, Pat voyagea notamment aux côtés de Marek Edelman avec lequel il se rendit dans un premier temps sur les ruines du ghetto de Varsovie $^{15}$. À cette occasion, le jeune bundiste remit à l'écrivain yiddish le journal rédigé en polonais en 1944 par un enfant juif alors âgé de treize ans et prénommé Henekh. Dans les pages d'un petit cahier, le garçon avait relaté son quotidien dans le ghetto de Varsovie, sa fuite à travers la

novembre 1946, JLC I, B 32 F 22, ainsi qu'une photo disponible dans la photothèque du Centre Medem - Arbeter Ring.

${ }^{12}$ Dans ses nombreux courriers adressés après-guerre aux bundistes parisiens, Yankev Pat ne tutoyait jamais ses interlocuteurs.

${ }^{13}$ Witold Bereś et Krzysztof Burnetko, Marek Edelman: Being on the Right Side, Redwood City, Bereś Media, 2015, p. 261. Cet ouvrage est une compilation de trois séries d'entretiens réalisés entre Marek Edelman et les journalistes Witold Bereś et Krzysztof Burnetko et publiés en polonais en 2008, 2009 et 2013.

${ }^{14}$ Yankev Pat, Ash un fayer: iber di khurves fun Poyln, New York, CYCO, 1946. Cet ouvrage fut traduit en anglais, dans une version quelque peu abrégée : Jacob Pat, Ashes and Fire: Through the Ruins of Poland, New York, International Universities Press, 1947.

${ }^{15}$ Il est fait mention de ces rencontres entre Pat et Edelman dans Khaim Shoshkes, Poyln 1946 : ayndrukn fun a rayze, Buenos Aires, Union centrale des Juifs polonais en Argentine, 1946, collection «Dos poylishe yidntum », vol. 8, p. 112-120 ainsi que dans Y. Pat, Ash un fayer..., op. cit., p. 15. 
campagne polonaise aux côtés de son frère Borukh, puis sa survie du côté aryen de Varsovie, son retour dans le ghetto, sa participation à son insurrection suivie de sa dispersion lors des derniers combats. Réfugié dans une maison des environs de Varsovie, il y avait rédigé son témoignage avant de périr lors de l'insurrection de la capitale polonaise. Yankev Pat affirma que dès sa première lecture des premières pages du manuscrit produit par Henekh, il avait réalisé qu'il tenait dans ses mains un véritable «trésor», le rare témoignage d'un enfant juif parmi les centaines de milliers d'entre eux qui furent assassinés, ce pourquoi il se serait décidé à le faire connaître en le publiant ${ }^{16}$.

C'est de ce « journal de bord » qu'il s'agissait lorsque le secrétaire général du JLC demanda à Edelman les noms des «deux jeunes qui [l'avaient] écrit ». Un seul enfant, Henekh, était en réalité l'auteur du cahier, son frère Borukh figurant cependant longuement dans le récit puisqu'il l'avait accompagné lors d'une de ses fuites du ghetto avant que sa trace ne soit perdue lors d'une sortie particulièrement périlleuse de ce dernier. Encore peu au fait de l'identité précise de l'auteur du journal, Pat n'en était alors sans doute en octobre 1946 qu'aux prémices de son travail de publication de ce texte, bien qu'il en ait déjà eu connaissance plusieurs mois auparavant. Souhaitant s'assurer qu'il allait obtenir le nom des auteurs, Pat réitéra sa demande à Edelman dans un télégramme envoyé dès le lendemain, le 9 octobre 1946, à la même adresse rue de la Mare $^{17}$. Le témoignage de Henekh, remanié en mêlant aux mots du garçon des précisions contextuelles et des commentaires personnels de Pat, parut finalement en yiddish en 1948 dans la collection Dos poylishe yidntum (La judéité polonaise) sous le titre Henekh, a yidish kind, vos iz aroys fun geto (Henekh, un enfant juif échappé du ghetto ${ }^{18}$.

Publiée de 1946 à 1966, cette collection dirigée par Marc Turkow depuis Buenos Aires édita 175 ouvrages comprenant des mémoires, fictions ou études sur la Shoah ou la vie juive avant et après-guerre en Pologne, parmi lesquelles se trouve le témoignage d'Élie Wiesel paru en $1956, \ldots$ Un di velt hot geshvign (... Et le monde se taisait), ouvrage dont les versions traduites et adaptées sont devenues célèbres, celle en

\footnotetext{
${ }^{16}$ Préface de Henekh, a yidish kind, vos iz aroys fun geto, Buenos Aires, Tsentralfarband fun poylishe yidn in Argentine, collection « Dos poylishe yidntum », vol. 29, 1948.

${ }^{17}$ Pat à Edelman, 9 octobre 1946, JLC I, B 32 F 22.

${ }^{18}$ Préface de Henekh..., op. cit. Henekh fut par la suite traduit en espagnol et en hébreu : Jacob Pat, Un nino judio salio del ghetto, Buenos Aires, Siglo Veinte, 1950 ; Id., Henekh, Tel Aviv, Y. L. Peretz, 1974.
} 
français étant parue en 1958 sous le titre La Nuit ${ }^{19}$. Euvre collective d'auteurs professionnels ou amateurs yiddishophones dispersés à travers le monde, Dos poylishe yidntum créait un lien mémoriel dans cette diaspora dont Yankev Pat fut l'un des maillons. Trace d'une multitude d'échanges entre les nombreux protagonistes impliqués dans cette entreprise éditoriale, la lettre de Pat nous permet à la fois d'observer une des étapes de la réalisation d'un des ouvrages de Dos poylishe yidntum et de savoir que le «jeune ami » lui ayant remis le cahier, mentionné par Pat dans sa préface de Henekh, était bien Marek Edelman ${ }^{20}$.
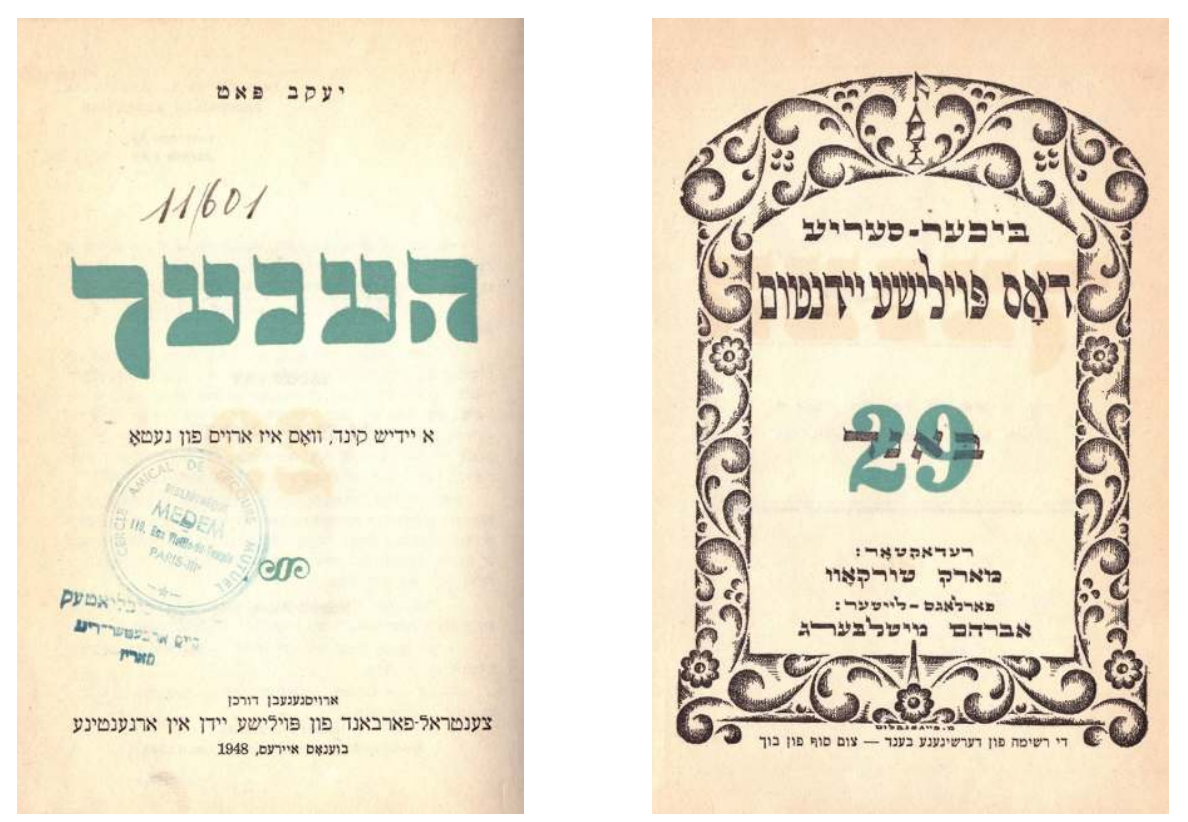

Page de titre et frontispice de Henekh, un enfant juif échappé du ghetto, ouvrage $\mathrm{n}^{\circ} 29$ de la collection Dos Poylishe yidntum. Sur la page de titre figure le tampon de la bibliothèque de la société de secours mutuel bundiste parisienne, l'Arbeter-ring.

\footnotetext{
${ }^{19}$ Paris, Les éditions de Minuit. Sur la collection Dos poylishe yidntum, voir, dans Judith Lindeberg (éd), Premiers savoirs de la Shoah..., op. cit.: Jan Schwarz, "Ashkénazes transnationaux: la culture yiddish après le génocide ", p. 231-243; Malena Chinski, « La correspondance de Marc Turkow et l'élaboration de la collection Dos poylishe yidntum », p. 245-255; J. Lindenberg, "Écrits d'histoire, écrits d'historiens dans la collection Dos poylishe yidntum», p. 257-271; J. Schwarz, Survivors and Exiles: Yiddish Culture after the Holocaust, Detroit, Wayne University Press, 2015, p. 92-117 ; Fleur Kuhn-Kennedy, « Mark Turkow et sa 'communauté imaginée' : l'activité éditoriale comme engagement intellectuel», Plurielles 19, 2015, p. 140-150.

${ }^{20}$ Y. Pat, Henekh..., op. cit., p. 5.
} 


\section{Quitter la Pologne?}

Après-guerre, Edelman ne joua pas uniquement un rôle de passeur entre l'enfant Henekh et l'écrivain Yankev Pat mais participa également à l'aide mise en place par le JLC en Pologne. D'après plusieurs correspondances entre New York, Varsovie et Łódź, le jeune bundiste se serait notamment impliqué dans l'aide aux enfants juifs orphelins et l'assistance à l'émigration juive de Pologne ${ }^{21}$, cette dernière action se trouvant confirmée par Edelman lors d'un entretien de la fin des années $2000^{22}$.

Encore peu d'actualité dans les mois suivant la Libération, la question de l'«exode de Pologne» (Yetsies Poyln) se posa surtout à partir de l'été 1946, dans un contexte de forte violence antisémite qui se manifesta en particulier dans les trains et lors de pogroms, le plus meurtrier étant celui perpétré à Kielce le 4 juillet $1946^{23}$. Au cours de cet été, la capitale française commença à devenir un lieu d'installation ou de transit, à la fois légal et illégal, de milliers de migrants juifs polonais faisant pour la plupart route vers des destinations outre-mer ${ }^{24}$. Le JLC et 1'Arbeter-ring parisien commencèrent alors à recevoir des centaines de demandes pressantes émanant de bundistes souhaitant quitter la Pologne. Parmi 8000 visas de transit obtenus par les organisations juives de France en août 1946, 300 furent délivrés aux bundistes, ceci en partie grâce à l'intervention de socialistes juifs français influents au sein de la Section française de 1'Internationale ouvrière (SFIO) comme du monde juif français et sollicités à ce sujet par le $\mathrm{JLC}^{25}$. Les bundistes qui

\footnotetext{
${ }^{21}$ Pat à Edelman, 13 octobre 1946 et 26 décembre 1946, JLC I, B 35 F 15 et 18 ; lettre non datée du JLC à Marek Edelman, classée dans un dossier pour janvier 1947, JLC I, B 35 F 19 ; Yoysef Brumberg à Edelman, 3 mars 1948, Jewish Labor Committee Records, Tamiment Library, Wagner Labor Archives, New York University, Part II (1948-1956), WAG.025.002 (désormais JLC II), B 93 F 1.

${ }^{22}$ W. Bereś et K. Burnetko, Marek Edelman: Being on the Right Side, op. cit., p. 291.

${ }^{23}$ Sur l'antisémitisme en Pologne après la Shoah, voir notamment Jan T. Gross, La peur : l'antisémitisme en Pologne après Auschwitz, Paris, Calmann-Lévy, 2010 [2006].

${ }^{24}$ D. Weinberg, «A Forgotten Postwar Jewish Migration...», op. cit.

${ }^{25}$ Sur l'obtention de visas d'immigration, voir Julia Maspero, « La politique française à l'égard de l'émigration juive polonaise de l'immédiat après-guerre », Bulletin du Centre de recherche français à Jérusalem 22, 2011 (en ligne : bcrfj.revues.org/6513). Sur l'aide des socialistes français aux bundistes au sujet de cette immigration, voir Adolph Held et Nathan Chanin à Daniel Mayer, 10 août 1946, et à André Blumel, 26 août 1946 ; Khil Najman à Yankev Pat, 6 septembre 1946, JLC I, B 32 F 21. En contrepartie de sa participation au financement du parti, le JLC recevait, dans des moments critiques, une aide pour son action d'aide à la reconstruction de la vie juive. Sur le financement du
} 
souhaitaient quitter la Pologne mirent cependant des semaines voire des années à franchir les obstacles administratifs et politiques à leur départ et beaucoup se résolurent à choisir la voie migratoire clandestine ${ }^{26}$. À l'exclusion des communistes, le JLC décida de venir en aide à chaque Juif polonais se tournant vers lui pour obtenir une assistance migratoire et de n'intervenir ni dans leurs choix de départ ni de destination ${ }^{27}$. La réaction de ses dirigeants face à cet «exode » ne fut toutefois pas constante. Initialement favorables aux efforts de reconstruction de la vie juive en Pologne, berceau du Bund et terre de leur enfance, Pat et ses collègues entrevoyaient encore, en 1946, un espoir d'avenir dans le monde juif rescapé de l'Est de l'Europe. Leur attitude changea cependant progressivement à mesure que se poursuivait la fermeture du « rideau de fer » et que les bundistes commençaient à être poursuivis en raison de leur appartenance politique. Courant 1947 et 1948, l' "émigration» (emigratsye) devint un «secours providentiel» (yeshue), le vocabulaire employé par le JLC évoluant parallèlement aux «persécutions » (gzeyres) politiques et difficultés croissantes de leurs camarades pour obtenir la «rédemption» (derleyzung) que représentait la sortie de Pologne ${ }^{28}$. En proie à des difficultés grandissantes pour intervenir dans un pays se vidant de ses rescapés juifs et se fermant à toute aide occidentale, le JLC se résolut à partir du printemps 1948 à concentrer ses activités de secours en Europe occidentale et en particulier en France ${ }^{29}$.

La lettre que Yankev Pat adressa en octobre 1946 à Marek Edelman démontre pourtant qu'avant même la mise en place du « rideau de fer», alors que le JLC s'impliquait quotidiennement en Pologne, entrevoyait encore un avenir juif dans ce pays et ne donnait pas priorité à l'effort d'émigration, son secrétaire général cherchait à convaincre l'ancien dirigeant de l'insurrection du ghetto de Varsovie de ne plus y

JLC à la SFIO, voir C. Pâris de Bollardière, «'La pérennité de notre peuple'... », op. cit., p. 365-433.

${ }^{26}$ Certains bundistes ayant entamé des démarches pour émigrer en 1946 n'arrivèrent en France qu'en 1957. Les parcours migratoires de ces bundistes sont détaillés dans les dossiers de suivi du Comité d'action sociale et de reconstruction (COJASOR), disponibles aux archives de cette organisation, cf. par exemple les dossiers SSI 42220 , SSI 42342 et SSI 41334.

${ }^{27}$ Le JLC vint notamment en aide à des centaines de sympathisants du parti sioniste de gauche Linke Poale Zion.

${ }^{28}$ À ce sujet, cf. C. Pâris de Bollardière, «'La pérennité de notre peuple'... », op. cit., p. 201-225.

${ }^{29}$ Benjamin Tabachinsky à Khil Najman, 25 mars 1948, JLC II, B 83 F 10. L'attention du JLC se déplaça aussi vers Israël, État dont il soutint la création tout en privilégiant l'aide en Europe. 
vivre. Ne considérant pas qu'il était possible «d'exploiter ses propres forces spirituelles » en Pologne « de la meilleure manière qu'il soit », Pat l'encourageait par conséquent à s'installer aux États-Unis afin d'y poursuivre des études puis d'y entamer sa vie professionnelle. Ce conseil prodigué "comme à un fils", qui tranchait avec les injonctions officielles de Pat et du JLC à ne pas influencer les Juifs de Pologne quant à leurs décisions migratoires, illustre les dilemmes plus intimes du dirigeant du JLC face à cette question fondamentale, pour nombre de Juifs originaires de Pologne favorables à la vie juive en diaspora, de continuer ou non à œuvrer au maintien de la vie juive dans leur pays natal en dépit des obstacles sociétaux et politiques qui mettaient ce projet en péril. S'il défendait encore de tels efforts, Yankev Pat souhaitait néanmoins, dès l'automne 1946, faire sortir de Pologne une personne qui lui était proche et dont il estimait qu'elle ne s'y épanouirait pas, commençant ainsi vraisemblablement à douter de la postérité du monde juif qui tentait de se reconstruire à l'Est de l'Europe. L'intervention de Pat auprès d'Edelman ne porta pas ses fruits. Au lendemain de Kielce, ce dernier s'arma pour riposter à des attaques antisémites dont il aurait pu être victime mais ne quitta pas la Pologne, où il avait déjà entamé des études de médecine dans la ville de Lódź $^{30}$. La démarche de Pat pour le persuader de partir, comme d'autres effectuées à cette époque en ce sens, n'y changèrent rien ${ }^{31}$. D'après ses souvenirs de la fin des années 2000, Edelman ne chercha pas en effet à rester à Paris à l'automne 1946 : «Mais Paris ne m'a pas attiré bien qu'il y avait des personnes qui disaient - restez, c'est une ville joyeuse. Une ville joyeuse ? Froide. Et au combien $»^{32}$. Bien que venu par la suite à Paris à plusieurs reprises, pour voir sa famille qui s'y installa au début des années 1970 ou pour des raisons politiques ou commémoratives, comme par exemple tenir un discours lors du centenaire du Bund en 1997, Edelman n'y résida jamais $^{33}$. Il demeura toute sa vie en Pologne, même lors de la vague antisémite de 1968 qui lui fit perdre son emploi de cardiologue dans un hôpital de Łódź. Il y est décédé à Varsovie le 2 octobre 2009.

\footnotetext{
${ }^{30}$ W. Bereś et K. Burnetko, Marek Edelman: Being on the Right Side, op. cit., p. 251$257 ; 261$.

${ }^{31}$ Selon un entretien d'Edelman, des tentatives pour le faire sortir de Pologne eurent aussi lieu en octobre 1945, cf. Ibid., p. 269-270.

${ }^{32}$ W. Bereś et K. Burnetko, Marek Edelman. Życie..., op. cit, p. 250.

${ }^{33}$ W. Bereś et K. Burnetko, Marek Edelman: Being on the Right Side, op. cit., p. 320323. Sur la présence de Marek Edelman au centenaire du Bund, voir les archives vidéo du Centre Medem-Arbeter Ring.
} 